\title{
Risk Factors for the Development of Bloodstream Infection in Patients Undergoing Haemodialysis in a Large Tertiary Hospital, Riyadh, Saudi Arabia
}

\section{Fahad Saleh Aldoghaim ${ }^{1 *}$, Norah Saleh Alnasyan ${ }^{2}$ and Abdurahman Nasser Alodayani ${ }^{1}$}

${ }^{1}$ Infection Control Department, Prince Sultan Military Medical City, Riyadh, Saudi Arabia

${ }^{2}$ College of Dentistry, Princess Nourah Bint Abdulrahman University, Riyadh, Saudi

Arabia

*Corresponding Author: Fahad Saleh Aldoghaim, Infection Control Department, Prince Sultan Military Medical City, Riyadh, Saudi Arabia.
Received: May 03, 2021

Published: June 12, 2021

(C) All rights are reserved by Fahad Saleh

Aldoghaim., et al.

\begin{abstract}
Introduction and Aim: Patients undergoing Haemodialysis are at high risk of developing bloodstream infection (BSI) because of their impaired immune defenses and repeated access of the bloodstream through vascular access sites. The major risk factor for the occurrence of haemodialysis-related BSI is the type of vascular access. The aim of this study was to evaluate the potential risk factors for the development of BSI in patients undergoing haemodialysis at Prince Sultan Medical Military City (PSMMC).

Methodology: Risk factors for the development of BSI in patients undergoing Haemodialysis was investigated using a retrospective case-control study with 1:1 matching conducted between September 2017 and September 2019. Cases were matched to controls by gender, age, time on hemodialysis and diagnosis of diabetes mellitus. Data were systematically collected from patient electronic medical records. Logistic regression was used for statistical analysis.

Results: The final study population included 120 patients (60 cases and 60 controls) were eligible to this study. There were no statistically significant differences between the two groups, except for the variables type of venous access and duration of venous access, where the p-value was $\leq 0.05$. Multiple logistic analysis showed that type of venous access was an independent risk factor for occurrence of BSI, where patients with a central venous catheters (CVC) were 7.75 times (CI 95\%: 2.65 - 22.66) more likely to develop BSI compared with patients who had an arteriovenous fistulas (AVF). Gram-positive bacteria were the most prevalent microorganisms isolated from the case group.

Conclusion: These findings emphasise the importance of specific measures for the insertion and maintenance of a CVC, particularly given that the number of patients that need haemodialysis treatment continues to grow in Saudi Arabia.
\end{abstract}

Keywords: Haemodialysis; Bloodstream Infection; Risk Factors; Arteriovenous Fistulas

\section{Background}

Haemodialysis continues to be one of the most popular medical treatments for end-stage renal disease (ESRD). Patients under- going haemodialysis are at high risk of developing a bloodstream infection (BSI) as a result their impaired immune defences and repeated access to the bloodstream through vascular access sites 
$[1,2]$. According to North American data, the BSI rates in patients on haemodialysis varies between 0.5 and 27.1 per 100 patients/ month, depending on the type of venous access used [3]. Further, the United States Renal Data System states that infection is the second leading cause of death in patients with ESRD [4].

Haemodialysis patients require a vascular access, which can be either a catheter or a graft. The major risk factor for the occurrence of haemodialysis-related BSI is the type of vascular access. Reports from previous studies have indicated that the infection risk is lowest when using arteriovenous fistulas (AVFs) and arteriovenous grafts (AVGs), and highest when using central venous catheters (CVCs) $[5,6]$. Furthermore, the prevalence of infection is high in the first six months of dialysis compared to the end of the first year [7]. Other identified risk factors for developing BSI include diabetes mellitus, anaemia, hypoalbuminaemia, female gender and methicillin-resistant Staphylococcus aureus (MRSA) nasal colonisation $[8,9]$.

According to the Saudi Centre for Organ Transplantation, a total of 16315 patients on haemodialysis therapy were recorded in 2016; and this number is expected to exceed 20000 patients in 2020 [10].

\section{Aim of the Study}

The aim of this study was to evaluate the incidence and the potential risk factors for the development of BSI in patients undergoing haemodialysis at Prince Sultan Medical Military City (PSMMC).

\section{Materials and Methods}

This was a retrospective, case-control study with 1:1 matching conducted between September 2017 and September 2019 at the haemodialysis unit at PSMMC. The unit serves an average of 350 haemodialysis patients/month.

Cases were defined as any patient over 18 years of age with ESRD treated with maintenance haemodialysis and suffering a BSI. BSI was defined as any positive blood culture according to the specific criteria of the National Healthcare Safety Network Dialysis Event Surveillance Manual (Centers for Disease Control and Prevention). Patients with two or more blood cultures sets of common commensal skin pathogens such as Corynebacterium spp. (not $C$. diphtheriae), Bacillus (not B. anthracis) spp., Propionibacterium spp., coagulase-negative staphylococci (including S. epidermidis), viridans group streptococci, Aerococcus spp. and Micrococcus spp. within time frame of 21 days were considered as having a BSI, or if the patient was treated for BSI by a physician. For patients who had more than one positive blood culture, only the first episode was considered for this study. Control subjects were selected from among the haemodialysis patients $\geq 18$ years of age at the same institution without positive blood cultures during the study period.

Cases were matched 1:1 to controls by gender, age, time on haemodialysis and diagnosis of diabetes mellitus. With regard to age, controls the same age as the case were selected, and if this was not possible, the closet age within two years was chosen. In the case of multiple acceptable controls, a random choice was made. Transient haemodialysis patients were excluded because they receive treatment from other dialysis providers.

\section{Statistical analysis}

The variables analysed for both case and control participants included patient demographic data, presence of diabetes mellitus and hypertension, type of vascular access, prior hospitalisation within three months and date of current vascular access. These data were systematically collected from patient electronic medical records. T-tests were used to examine the associations between the quantitative variables and the presence of BSI. The chi-square test of significance was used to examine the associations between the primary outcome (BSI) and categorical variables. A logistic regression model was used to compute the odds ratio with confidence intervals of $95 \%$.

\section{Results}

A total of 315 patients undergoing renal replacement therapy between September 2017 and September 2019 at PSMMC were screened, of whom $64(20.32 \%)$ met the inclusion criteria. Four patients were excluded as they were unable to be paired with a control. The final study population included 120 patients ( 60 cases and 60 controls). The demographic characteristics and the clinical variables of interest are presented in table 1 . There were no statistically significant differences between the two groups, except for the variables type of venous access and duration of venous access, where the $p$-value was $\leq 0.05$. 
Table 1: Demographic characteristics and clinical variables of interest of the cases and controls.

\begin{tabular}{|c|c|c|c|}
\hline & Case $(n=60)$ & Control $(n=60)$ & $P$-value \\
\hline Age & $64.24 \pm 17.284$ & $63.79 \pm 17.09$ & 0.8865 \\
\hline \multicolumn{4}{|l|}{ Sex } \\
\hline Male & $35(58.33)$ & $35(58.33)$ & \multirow{2}{*}{0.453} \\
\hline Female & $25(41.67)$ & $25(41.67)$ & \\
\hline \multicolumn{4}{|l|}{ Comorbidities } \\
\hline diabetes mellitus & $41(68.33)$ & $41(68.33)$ & 0.845 \\
\hline HTN & $51(85)$ & $58(96.67)$ & 0.044 \\
\hline \multicolumn{4}{|c|}{ Type of venous access } \\
\hline FPC & $5(8.33)$ & $1(1.67)$ & 0.094 \\
\hline PCRJ & $25(41.67)$ & $18(30)$ & 0.183 \\
\hline PCLJ & $12(20)$ & $6(10)$ & 0.125 \\
\hline PCRS & $10(16.67)$ & $7(11.66)$ & 0.432 \\
\hline PCLS & $3(5)$ & $1(1.67)$ & 0.309 \\
\hline AVF & $5(8.33)$ & $27(45.00)$ & $<0.001$ \\
\hline \multicolumn{4}{|c|}{ Duration of venous access } \\
\hline $0-30$ days & $9(15.00)$ & $4(7.00)$ & 0.142 \\
\hline $30-180$ days & $18(30.00)$ & $9(15)$ & 0.049 \\
\hline$>180$ days & $33(55.00)$ & $47(78.00)$ & 0.007 \\
\hline $\begin{array}{l}\text { Previous } \\
\text { hospitalization }\end{array}$ & $32(53.3)$ & $45(75)$ & 0.013 \\
\hline
\end{tabular}

Values are expressed as mean \pm SD or $\mathrm{n}(\%)$; HTN: Hypertension; FPC: Femoral Permanent Catheter; PCRJ: Permanent Catheter Right Jugular; PCLJ: Permanent Catheter Left Jugular; PCRS: Permanent Catheter Right Subclavian; PCLS: Permanent Catheter Left Subclavian; AVF: Arteriovenous Fistula.

The results of the logistic regression are presented in table 2 . In simple logistic regression, type of venous access and duration of venous access were significantly associated with the occurrence of BSI. In the multiple logistic regression, type of venous access was an independent risk factor for occurrence of BSI, where patients with a CVC were 7.75 times (CI 95\%: 2.65-22.66) more likely to develop BSI compared with patients who had an AVF.
Table 2: Simple and multiple logistic regression analyses.

\begin{tabular}{|c|c|c|}
\hline & $\begin{array}{c}\text { Simple logistic } \\
\text { regression }\end{array}$ & $\begin{array}{c}\text { Multiple logistic } \\
\text { regression }\end{array}$ \\
\hline $\begin{array}{l}\text { Diagnosis of } \\
\text { hypertension }\end{array}$ & $\begin{array}{c}0.22(0.04,1.08) \\
0.063\end{array}$ & $\begin{array}{c}0.30(0.06,1.62) \\
0.164\end{array}$ \\
\hline \multicolumn{3}{|l|}{$\begin{array}{l}\text { Type of venous } \\
\text { access }\end{array}$} \\
\hline FPC vs AVF & $\begin{array}{c}27.00(2.58 \\
282.98) ; 0.006\end{array}$ & \\
\hline CVC vs AVF & $\begin{array}{c}9.00(3.16, \\
25.65) ;<0.001\end{array}$ & $\begin{array}{c}7.75(2.65,22.66) ; \\
<0.001\end{array}$ \\
\hline \multicolumn{3}{|l|}{$\begin{array}{l}\text { Duration of } \\
\text { venous access }\end{array}$} \\
\hline $\begin{array}{l}0-30 \text { vs }>180 \\
\text { days }\end{array}$ & $\begin{array}{c}3.20(0.91 \\
11.29) ; 0.070\end{array}$ & $\begin{array}{c}2.25(0.64,10.18) ; \\
0.184\end{array}$ \\
\hline $\begin{array}{l}30-180 \text { vs }>180 \\
\text { days }\end{array}$ & $\begin{array}{c}2.85(1.14,7.12) ; \\
0.025\end{array}$ & $\begin{array}{c}2.11(0.78,5.71) \\
0.140\end{array}$ \\
\hline
\end{tabular}

CVC: Central Venous Catheter; FPC: Femoral Permanent Catheter; AVF: Arteriovenous Fistula.

Gram-positive bacteria were the most prevalent microorganisms isolated from the case group. The most frequently isolated gram-positive bacteria were coagulase-negative Staphylococcus (31.7\%) followed by Staphylococcus aureus (16.7\%) and Enterococcus faecalis (5.0\%). Enterobacter cloacae was the most frequently isolated gram-negative species isolated in blood cultures from the case group (15\%). Resistant bacteria were isolated from seven patients: four cases with MRSA, two with extended spectrum beta-lactamase resistant Klebsiella pneumoniae and one patient with multi-resistant Chryseobacterium meningosepticum which resistance to amikacin, gentamycin, ceftriaxone, imipenem and meropenem (Table 3). 
Table 3: Microorganisms isolated and profiles of resistance.

\begin{tabular}{lc}
\hline & Total \\
\hline Gram-positives & $\mathbf{3 3} \mathbf{( 5 5 \% )}$ \\
Staphylococcus aureus & $6(18.2)$ \\
Methicillin-resistent S aureus & $4(12.1)$ \\
Staphylococcus coagulase negative & $19(57.6)$ \\
Enterococcus faecalis & $3(9.1)$ \\
Viridans streptococcus & $1(3)$ \\
Gram-negative & $\mathbf{2 7}(\mathbf{4 5 \% )}$ \\
Enterobacter cloacae & $9(33.3)$ \\
Pseudomonas aeruginosa & $5(18.5)$ \\
Klebsiella pneumoniae & $2(7.4)$ \\
ESBL Klebsiella pneumoniae & $2(7.4)$ \\
Pantoea species (pantoea agglomerans) & $3(11.1)$ \\
Serratia marcescens & $2(7.4)$ \\
Stenotrophomonas maltophilia & $1(3.7)$ \\
Acinetobacter baumanii & $1(3.7)$ \\
Salmonella enteriditis & $1(3.7)$ \\
MR Chryseobacterium meningosepticum & $1(3.7)$ \\
Total of microrganismos & $\mathbf{6 0}$ \\
\hline
\end{tabular}

Values are expressed as n (\%). MR: multi-resistant.

\section{Discussion}

The major risk factors associated with BSI are the type of vascular access, previous hospitalisation, female gender and diabetes mellitus $[8,9]$. The present study evaluated the risk factors for developing BSI in patients undergoing renal replacement therapy at PSMMC. In our study, the use of CVC and FPC was found to be associated with the development of BSI (OR: 7.75; CI 95\%: 2.65, 22.66; $\mathrm{p}<0.001$ ) compared with the use of AVF. This result is in agreement with other findings showing higher rates of BSI among haemodialysis patients with CVCs compared to patients with AVFs [12,13]. Dayana., et al. (2015) found that haemodialysis patients using CVC had an 11.2-fold higher risk of developing BSI compared to patients using AVF. Furthermore, a cohort study conducted by Xui., et al. (2013) showed that the occurrence of BSI in haemodialysis patients was three times higher when CVC used. A retrospective study from a university hospital in Greece found that BSI was lowest when using AVFs and AVGs and highest when using CVCs (OR:
2.93; $\mathrm{p}=0.047)[9]$.

Duration of venous access is also a potential risk factor for BSI. In previous studies, the majority of BSIs were found to be associated within 90 days of first insertion of the venous access [12,14]. However, in the current study three categories were used to analyse the duration of venous access (0-30 days, 30-180 days, and $>180$ days), and BSI was found to be associated with venous access inserted more than 180 days earlier. This may be the result of the successful use of aseptic technique during the venous access insertion procedure and the implementation of care bundles.

With regards to the microorganisms isolated, previous studies have reported a high prevalence of gram-positive bacteria $[9,12,15]$. These results are in accordance with the current study, where gram-positive organisms were the predominant isolates. Coagulase-negative species (CNSS) of Staphylococcus were the most common bacteria isolated (31.7\%), followed by Staphylococcus aureus (16.7\%) and Enterococcus faecalis (5.0\%). However, this result differs to those of other studies, which have shown $S$. aureus was the most frequently isolated gram-positive bacterial species $[9,12,15]$. The high prevalence of CNSS in our study may result from the predominant colonisation of the internal catheter surface from the skin. Christoph., et al. (2005) reported that significant colonisation by CNSS appeared around three weeks after catheter insertion. Despite the predominance of gram-positive organisms in the present study, gram-negative bacteria accounted for $45 \%$ of the species isolated.

Patients receiving dialysis are also at high risk of developing infection caused by resistant organisms, due to their frequent hospitalisations and need for antimicrobial treatment. A retrospective study was contacted in Jeddah, Saudi Arabia demonstrated that more than a third of bacteria isolated from haemodialysis patients were resistant organisms [11]. In contrast to this, the proportion of resistant pathogens identified in our study was only $11.7 \%(7 / 60)$ of all isolates.

It has recently been demonstrated that female gender, previous hospitalisation and colonisation by MRSA are risk factors for the development of BSI in haemodialysis patients [9,12,14]. However, in our study, we did not observe any associations between these risk factors and the development of BSI. 
The present study has several limitations. First, it was a retrospective study, and missing data might have concealed potential risk factors that were not documented in the medical records. Additionally, the study was performed in one hospital in single geographic area, and thus others centres and geographic areas of the Kingdom of Saudi Arabia should be involved to present a more comprehensive view.

\section{Conclusion}

A major risk factor for BSI in patients undergoing haemodialysis is still the use of a CVC. The use of an AVF is the best available option to reduce the risk BSI relative to the use of CVCs. Gram-positive bacteria were the most prevalent organisms isolated in our study, of which $13.8 \%$ were resistant. Our study emphasises the importance of specific measures for the insertion and maintenance of a CVC, particularly given that the number of patients that need haemodialysis treatment continues to grow in Saudi Arabia.

\section{Bibliography}

1. Hörl WH. "Neutrophil function and infections in uremia". American Journal of Kidney Diseases 33.2 (1999): xlv-xlviii.]

2. Jaber BL. "Bacterial infections in hemodialysis patients: pathogenesis and prevention". Kidney International 67 (2005): 25082519.

3. Klevens RM., et al. "Dialysis Surveillance Report: National Healthcare Safety Network (NHSN)-data summary for 2006". Seminars in Dialysis 21 (2008): 24-28.]

4. Saran R., et al. "US renal data system 2016 annual data report: epidemiology of kidney disease in the United States". American Journal of Kidney Diseases 69 (2017): A7-A8.'

5. Churchill DN., et al. "Canadian hemodialysis morbidity study". American Journal of Kidney Diseases 19 (1992): 214-234.]

6. Hoen B., et al. "EPIBACDIAL: a multicenter prospective study of risk factors for bacteremia in chronic hemodialysis patients". Journal of the American Society of Nephrology 9 (1998): 869876.

7. Moor CL., et al. "Comparative effectiveness of two catheter locking solutions to reduce catheter-related bloodstream infection in hemodialysis patients". Clinical Journal of the American Society of Nephrology 9 (2014): 1232-1239.
8. Nguyen DB., et al. "Invasive methicillin-resistant Staphylococcus aureus infections among patients on chronic dialysis in the United States, 2005-2011". Clinical Infectious Diseases 57 (2013): 1393-1400.

9. Fysaraki M., et al. "Incidence, clinical, microbiological features and outcome of bloodstream infections in patients undergoing hemodialysis". International Journal of Medical Sciences 10 (2013): 1632-1638.

10. Saudi Center for Organ Transplantation. Annual Report 2016 Hemodialysis in the Kingdom of Saudi Arabia (2018).

11. Alhazmi SM., et al. "Bloodstream infection at hemodialysis facilities in Jeddah: a medical record review". Annals of Saudi medicine 39 (2019): 258-264.

12. Fram D., et al. "Risk factors for bloodstream infection in patients at a Brazilian hemodialysis center: a case-control study". BMC Infectious Diseases 15 (2015): 158.]

13. Xue H., et al. "Hemodialysis access usage patterns in the incident dialysis year andassociated catheter-related complications”. American Journal of Kidney Diseases 61 (2013): 123-130.

14. Napalkov P., et al. "Incidence of catheter-related complications in patients with central venous or hemodialysis catheters: a health care claims database analysis". BMC Cardiovascular Disorder 13 (2013): 86.

15. Gauna TT., et al. "Bloodstream infection in patients with endstage renal disease in a teaching hospital in central-western Brazil". Revista da Sociedade Brasileira de Medicina Tropical 46 (2013): 426-432.

\section{Volume 5 Issue 7 July 2021}

(C) All rights are reserved by Fahad Saleh Aldoghaim., et al. 